\title{
PROJETO DE UM CONTROLADOR PID ROBUSTO PARA UM SISTEMA DE DOIS TANQUES
}

\author{
TASSALON FERREIRA DA SILVA ${ }^{1}$, MÁRCIA LISSANDRA MACHADO PRADO ${ }^{1}$.
}

\author{
1. Laboratório de Sistemas de Controle e Robótica, Departamento de Tecnologia, Universidade Estadual \\ de Feira de Santana-BA \\ CEP: 44036-900, Avenida Transnordestina, S/N - Novo Horizonte - Feira de Santana - Bahia \\ E-mails: tassalonfs@gmail.com, marcia.lissandra@gmail.com
}

\begin{abstract}
This paper exhibits the proposed the design of a robust controller for the system of two tanks of the Quanser, using to this, concepts of modal interval analysis and then presents the main results. It also makes comparisons of the controllers developed with traditional control techniques to assess the results and validate the advantages of the use of robust controllers. The results and comparisons are performed in simulation environments using the MATLAB that can model and simulate a wide variety of control systems.
\end{abstract}

Keywords— Control Systems: Robust Control: Modals Intervals: Two Tanks System.

Resumo - Este trabalho propõe o projeto de um controlador robusto para o Sistema de Dois Tanques Acoplados da Quanser usando conceitos de análise intervalar. Também são realizadas comparações do controlador desenvolvido com técnicas de controle tradicionais a fim de avaliar os resultados e validar as vantagens do uso de controladores robustos. Os resultados e as comparações são realizados em ambientes de simulação usando o software MATLAB que permite de forma ampla modelar e simular os mais variados sistemas de controle.

Palavras-chave— Sistema de Controle, Controle Robusto, Intervalos Modais, Sistema de Dois Tanques Acoplados.

\section{Introdução}

Os sistemas de controle são utilizados nas mais diversas áreas da indústria, se tornando essenciais em qualquer campo da engenharia e da ciência (Ogata, 2010). Exemplos de sua utilização estão em sistemas robóticos, veículos espaciais e em operações industriais que envolvam o controle de temperatura, vazão, pressão, etc (Ogata, 2010).

A teoria de controle robusto é utilizada quando se deseja considerar a existência de incertezas ou erros entre a planta real e o seu modelo matemático no projeto do controlador (Ogata, 2010). Esta teoria considera que o sistema físico a ser controlado e o ambiente onde irá operar não pode ser modelado com exatidão. Há também a possibilidade de interferências por meio de ruídos em sensores e perturbações imprevisíveis. Isso gera valores inesperados que o controlador deve ser capaz de tratar. Para o projeto de controladores altamente precisos mesmo na presença de consideráveis incertezas é conveniente recorrer a sistemas de controle robustos (Dorf, 2001).

O controlador robusto proposto neste trabalho será projetado com base nas variações dos parâmetros do sistema utilizando, para isso, intervalos modais. A substituição de valores pontuais dos parâmetros do sistema por intervalos permite tornar os parâmetros do sistema variáveis e com isso, usando a aritmética intervalar modal, é possível realizar o projeto do controlador robusto.

A análise intervalar modal (Gardenes et al, 2001) é uma alternativa à análise intervalar clássica (Moore, 1979; Acióly, 1991), que pode contornar o problema de sobre-estimação dos intervalos resultantes do projeto de controladores robustos (Prado e Paz, 2008).

A planta para a qual o controlador a ser desenvolvido será utilizada é o Sistema de Dois Tanques da Quanser (Quanser, 2004), um sistema hidráulico, onde muitas das suas configurações são aplicadas na indústria. Tratando-se de um sistema didaticamente adequado para fins de projeto de controladores.

Neste artigo, no item Revisão da Literatura, serão apresentados os principais conceitos relacionados aos projetos de sistemas de controle robusto usando Análise Intervalar Modal. No item Metodologia são mostrados detalhes da modelagem do Sistema de Dois Tanques da Quanser, enfocando as suas principais características, e é apresentado também o controlador PID Robusto desenvolvido para o sistema modelado, usando os conceitos de Análise Intervalar Modal. Em Resultados são apresentados os testes realizados e comparações com outros projetos de controladores. Por fim, são feitas algumas conclusões dos resultados obtidos no item Conclusão.

\section{Revisão da Literatura}

\subsection{Análise intervalar Clássica}

A teoria matemática de análise intervalar busca melhorar a exatidão e eficiência que há na prática da computação científica (Acióly, 1991). Métodos convencionais trabalham em cima de uma estimativa para uma resposta junto a um erro associado, porém uma análise precisa do erro é custosa e nem sempre viável (Acióly, 1991). 
Técnicas intervalares garantem que as respostas pertençam a intervalos, promovendo maior segurança na precisão dos resultados. Uma resposta intervalar tem a garantia de sua incerteza, diferente de outros métodos que resultam apenas em uma estimativa do erro (Acióly, 1991). como:

O conjunto dos números intervalares é definido

$$
I(\mathbb{R}):=\{[\mathrm{a}, \mathrm{b}] \mid \mathrm{a} \in \mathbb{R}, \mathrm{b} \in \mathbb{R}, \mathrm{a} \leq \mathrm{b}\}
$$

Ou seja, um número intervalar [x]' é composto por um par ordenado de números reais $\left[\mathrm{x}_{1}, \mathrm{x}_{2}\right]$ que delimita o intervalo de [x]' onde $\mathrm{x}_{1} \leq \mathrm{x}_{2}$, sendo que $\mathrm{x}_{1}$ e $\mathrm{x}_{2}$ são os extremos do intervalo [x]'.

Análise intervalar clássica apresenta sobreestimação nos resultados de cálculos intervalares que podem ser resolvidos usando intervalos modais (Gardenes et al, 2001) e (Prado, 2006).

\subsection{Análise intervalar Modal}

Os intervalos modais consistem em um par formado por um intervalo clássico e um quantificador, e sua representação pode ser vista a seguir (Gardenes et al, 2001).

$$
[\mathrm{x}]:=\left([\mathrm{x}]^{\prime}, \mathrm{Q}[\mathrm{x}]\right)
$$

$\mathrm{O}$ quantificador $\mathrm{Q}[\mathrm{x}]$ indica a modalidade de um intervalo que pode ser existencial $(\mathrm{E})$, quando existe um $\mathrm{x} \in[\mathrm{x}]$ ', ou universal (U), para todo $\mathrm{x} \epsilon$ $[\mathrm{x}]$ '.

Predicados que são condições que podem ser associadas a um intervalo. Portanto, na análise intervalar modal um intervalo é identificado pelos elementos do seu intervalo clássico que satisfazem à condição imposta pelo predicado associado (Gardenes et al, 2001). Um exemplo da notação utilizada para relacionar quantificadores a predicados é:

$$
E\left(\mathrm{x},[\mathrm{a}]{ }^{\prime}\right) \mathrm{P}(\mathrm{x})
$$

Isso nos diz que existe um $\mathrm{x} \in[\mathrm{a}]$ ' tal que o predicado $\mathrm{P}(\mathrm{x})$ é satisfeito. $\mathrm{O}$ conjunto dos intervalos modais pode então ser representado por:

$$
I^{*}(\mathbb{R}):=\left\{\left([\mathrm{x}]^{\prime},\{\mathrm{E}, \mathrm{U}\}\right) \mid[\mathrm{x}]^{\prime} \in \mathrm{I}(\mathbb{R})\right\}
$$

Quando, em um intervalo $[\mathrm{x}]=\left[\mathrm{x}_{1}, \mathrm{x}_{2}\right], \mathrm{x}_{1} \leq \mathrm{x}_{2}$, $\mathrm{o}$ intervalo pode ser chamado de próprio (e universal), caso contrário, ele é chamado de intervalo impróprio (e existencial).

Algumas operações aritméticas intervalares podem ser vistas a seguir. Considerando os intervalos $\mathrm{A}$ $=\left[a_{1}, a_{2}\right]$ e $B=\left[b_{1}, b_{2}\right]$, onde $a_{1}$ e $a_{2}$ são os extremos do intervalo $A$, e $b_{1}$ e $b_{2}$ são os extremos do intervalo $\mathrm{B}$, temos que:

$$
\begin{gathered}
A+B=\left[a_{1}+b_{1}, a_{2}+b_{2}\right] \\
A-B=\left[a_{1}-b_{2}, a_{2}-b_{1}\right] \\
A * B=\left[a_{1} b_{1}, a_{2} b_{2}\right] \text { para } a_{1}, a_{2}, b_{1}, b_{2} \geq 0
\end{gathered}
$$

$$
\begin{gathered}
A / B=\left[a_{1} / b_{2}, a_{2} / b_{1}\right] \text { para } a_{1}, a_{2} \geq 0 \text { e } b_{1}, b_{2}>0 \\
\operatorname{dual}(A)=\left[a_{2}, a_{1}\right]
\end{gathered}
$$

Existem diversas operações e propriedades que são aplicadas a intervalos modais. Os detalhes das propriedades e operações aritméticas intervalares, podem ser encontrados em (Gardenes et al, 2001) e (Group, 1998). Para os casos onde são usados vetores e matrizes as definições são equivalentes.

Considerando $f$ uma função contínua de $\mathbb{R}^{\mathrm{n}}$ para $\mathbb{R}$, chama-se de extensão intervalar modal de $f$, sobre o intervalo [a], qualquer função $F$ de $I^{*}\left(\mathbb{R}^{\mathrm{n}}\right)$ para o $I *(\mathbb{R})$ que satisfaz a propriedade (Prado, 2006):

$$
\begin{gathered}
\left.\mathrm{U}\left([\mathrm{x}]^{\prime}, \mathrm{I}\left(\mathbb{R}^{\mathrm{n}}\right)\right)\left(\left(\mathrm{x} \in[\mathrm{x}]^{\prime}\right) \in \mathrm{P}^{*}([\mathrm{a}])\right)\right)\left(\mathrm{f}(\mathrm{x}) \in \mathrm{f}\left([\mathrm{x}]^{\prime}\right)\right) \\
\in \mathrm{P}^{*}(\mathrm{~F}([\mathrm{a}]))
\end{gathered}
$$

Considerando a função $f$ contínua de $\mathbb{R}^{\mathrm{n}}$ em $\mathbb{R}$, [a] $\in I^{*}\left(\mathbb{R}^{\mathrm{n}}\right)$, e $\left(\mathrm{a}_{\mathrm{p}}, \mathrm{a}_{\mathrm{i}}\right)$ componentes de separação de $[\mathrm{a}]=\left(\left[\mathrm{a}_{\mathrm{p}}\right],\left[\mathrm{a}_{\mathrm{i}}\right]\right)$, com $\left[\mathrm{a}_{\mathrm{p}}\right]$ um subvetor contendo as componentes próprias de $[\mathrm{a}]$ e $\left[\mathrm{a}_{\mathrm{i}}\right]$ um subvetor contendo as componentes impróprias de [a]. Definem-se as extensões intervalares semânticas modais $f^{*}$ e $f^{* *}$ pelas expressões abaixo (Prado, 2006).

$$
\begin{aligned}
& f^{*}([a])=\left[{ }^{m i n} a_{p} \in\left[a_{p}\right]^{\prime}\left({ }^{m a x} a_{i} \in\left[a_{i}\right]^{\prime}\left(f\left(a_{p}, a_{i}\right)\right)\right),{ }^{\max } a_{p} \epsilon\right. \\
& \left.\left[a_{p}\right] \text { ' }\left({ }^{\min } a_{i} \in\left[a_{i}\right]^{\prime}\left(f\left(a_{p}, a_{i}\right)\right)\right)\right]
\end{aligned}
$$

$f^{* *}([a])=\left[{ }^{\max } a_{i} \in\left[a_{i}\right]^{\prime}\left({ }^{\min } a_{p} \in\left[a_{p}\right]^{\prime}\left(f\left(a_{p}, a_{i}\right)\right)\right),{ }^{\min } a_{i} \epsilon\right.$ $\left.\left[a_{i}\right]^{\prime}\left({ }^{\max } a_{p} \in\left[a_{p}\right]^{\prime}\left(f\left(a_{p}, a_{i}\right)\right)\right)\right]$

Pode-se chamar de unimodal uma função $f$, em um intervalo [a], se ela for totalmente monótona em todo o seu intervalo, ou seja, se $f$ for totalmente crescente ou decrescente em [a]. Quando a função é unimodal para o intervalo [a], as extensões são iguais. Além disso, caso [a] seja um intervalo próprio, $f^{*}$ é a imagem de $f$ no domínio [a]'. Porém, nem sempre $f^{*}$ e $f^{* *}$ podem ser calculadas diretamente (Prado, 2006).

Os predicados dos resultados de cálculos intervalares podem ser atribuídos utilizando as extensões semânticas. Para isso, são necessários dois teoremas semânticos. Eles fornecem um significado completo dos cálculos intervalares (Prado, 2006). Considere $F$ : $I^{*}\left(\mathbb{R}^{\mathrm{n}}\right) \rightarrow I^{*}(\mathbb{R})$.

Teorema 1 - Teorema Semântico para $f^{*}$ (Group, 1998). Se [a] $\in I^{*}\left(\mathbb{R}^{\mathrm{n}}\right), f$ é contínua em [a]' e $\mathrm{F}([\mathrm{a}]) \in I^{*}(\mathbb{R})$, então $f^{*}([\mathrm{a}]) \subseteq \mathrm{F}([\mathrm{a}])$ se e somente se:

$$
\mathrm{U}\left(\mathrm{a}_{\mathrm{p}},\left[\mathrm{a}_{\mathrm{p}}\right]^{\prime}\right) \mathrm{Q}(\mathrm{z}, \mathrm{F}([\mathrm{a}])) \mathrm{E}\left(\mathrm{a}_{\mathrm{i}},\left[\mathrm{a}_{\mathrm{i}}\right]^{\prime}\right)\left(\mathrm{z}=\mathrm{f}\left(\mathrm{a}_{\mathrm{p}} ; \mathrm{a}_{\mathrm{i}}\right)\right) \text {. }
$$

Teorema 2 - Teorema Semântico para $f^{* *}$ (GROUP, 1998). Se [a] $\in I^{*}\left(\mathbb{R}^{\mathrm{n}}\right), f$ é contínua em [a]' e $\mathrm{F}([\mathrm{a}]) \in I^{*}(\mathbb{R})$, então $f^{* *}([\mathrm{a}]) \supseteq \mathrm{F}([\mathrm{a}])$ se e somente se:

$\mathrm{U}\left(\mathrm{a}_{\mathrm{i}},\left[\mathrm{a}_{\mathrm{i}}\right]^{\prime}\right) \mathrm{Q}(\mathrm{z}, \operatorname{Dual}(\mathrm{F}([\mathrm{a}]))) \mathrm{E}\left(\mathrm{a}_{\mathrm{p}},\left[\mathrm{a}_{\mathrm{p}}\right]^{\prime}\right)\left(\mathrm{z}=\mathrm{f}\left(\mathrm{a}_{\mathrm{p}}, \mathrm{a}_{\mathrm{i}}\right)\right)$. 
Teorema 3 (Group, 1998). Seja [x] um vetor intervalar, $f \mathrm{R}$ definida no domínio de [x]' e totalmente monótona para todos os seus componentes multiincidentes, ou seja, todos os componentes que aparecem mais de uma vez. Seja [xd] o vetor estendido de [x], tal que cada incidência de todo componente multi-incidente seja incluído em [xd] como um componente independente, mas transformado em seu dual se o correspondente ponto incidente tiver monotonicidade no sentido contrário ao seu global do correspondente componente de [x]. Então,

$$
f^{*}([\mathrm{x}])=f \mathrm{R}([\mathrm{xd}])=f^{* *}([\mathrm{x}])
$$

A monotonicidade das incidências dos componentes pode ser investigada utilizando derivadas parciais.

\section{Metodologia}

\subsection{A Modelagem do Sistema de Dois Tanques}

O Sistema de Dois Tanques da Quanser é um protótipo de simulação de processos, usa dois tanques interligados em cascata. O fluxo contínuo é regulado por uma bomba e não tem válvulas de controle. A água é bombeada para um ou para os dois tanques, conforme a configuração feita, por meio de tubos com dimensão especifica. Os dois tanques deste sistema são montados de forma que o fluxo de saída do primeiro tanque flui para dentro do segundo tanque, e o fluxo de saída do segundo tanque flui para dentro do reservatório (Quanser, 2004).

A configuração do Sistema de Dois Tanques adotada para este trabalho resulta na alimentação do primeiro tanque por meio da bomba e o segundo tanque alimentado somente pelo fluxo de saída do primeiro tanque como pode ser visualizado na Figura 1. $L 1$ e $L 2$ representam os níveis de água do primeiro e do segundo tanque, respectivamente. A variável a ser controlada é o nível de água do segundo tanque, ou seja, $L 2$.

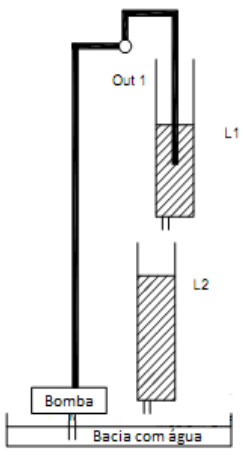

Figura 1. Configuração do Sistema de Dois Tanques para o projeto do controlador.

O Sistema de Dois Tanques da Quanser fornece informações suficientes a respeito da planta para que possa ser modelada conforme a configuração adota- da. Pelo fato das equações que regem o sistema não serem lineares, é necessário linearizá-las sobre um ponto de operação $\mathrm{L}_{10}$, para o primeiro tanque, e $\mathrm{L}_{20}$, para o segundo tanque, usando a série de Taylor (Ogata, 2010). Após a linearização do sistema, as variações nos níveis do primeiro e segundo tanque são representadas por (1) e (2).

$$
\begin{gathered}
L_{1}^{\prime}=-\frac{a_{1}}{A_{1}} \sqrt{\frac{g}{2 L_{10}}} L_{1}+\frac{K_{m}}{A_{1}} V_{p} \mathrm{~cm} / \mathrm{s} \\
L_{2}^{\prime}=-\frac{a_{2}}{A_{2}} \sqrt{\frac{g}{2 L_{20}}} L_{2}+\frac{a_{1}}{A_{2}} \sqrt{\frac{g}{2 L_{10}}} L_{1} \mathrm{~cm} / \mathrm{s}
\end{gathered}
$$

Onde $a_{1}$ e $a_{2}$ são os diâmetros dos orifícios do primeiro e segundo tanque, respectivamente. $\mathrm{A}_{1}$ e $\mathrm{A}_{2}$ são as áreas do primeiro e segundo tanque. Os valores de $A_{1}, A_{2}, a_{1}$ e $a_{2}$ podem ser alterados na planta para as diversas opções disponíveis. $E V_{p}$ é a tensão aplicada à bomba.

Para realizar a modelagem do sistema no software de simulação Matlab, adotaram-se os valores padrões especificados pelo fabricante. A Tabela 1 mostra os valores de cada variável envolvida na modelagem do Sistema de Dois Tanques.

Tabela 1. Valores utilizados na modelagem do sistema.

\begin{tabular}{|l|l|l|c|}
\hline Variável & \multicolumn{1}{|c|}{ Valor } & Variável & Valor \\
\hline $\mathrm{A}_{1}$ & $15,5179 \mathrm{~cm}^{2}$ & $\mathrm{~K}_{\mathrm{m}}$ & $4,6\left(\mathrm{~cm}^{3} / \mathrm{s}\right) / \mathrm{Volt}$ \\
\hline $\mathrm{A}_{2}$ & $15,5179 \mathrm{~cm}^{2}$ & $\mathrm{~g}$ & $980 \mathrm{~cm}^{2} / \mathrm{s}$ \\
\hline $\mathrm{a}_{1}$ & $0,17813919 \mathrm{~cm}$ & $\mathrm{~L}_{10}$ & $15 \mathrm{~cm}$ \\
\hline $\mathrm{a}_{2}$ & $0,17813919 \mathrm{~cm}$ & $\mathrm{~L}_{20}$ & $15 \mathrm{~cm}$ \\
\hline
\end{tabular}

A partir das equações apresentadas anteriormente para $L_{1}{ }_{1}$ e $L_{2}$, é possível encontrar a função de transferência do sistema completo (3).

$$
\frac{L_{2}(s)}{V_{p}(s)}=\frac{0,0194}{s^{2}+0,1312 s+0,0043}
$$

Neste projeto, a variável que se pretende controlar é o nível de água do segundo tanque. Quando o sistema torna-se realimentado, com realimentação unitária, percebe-se uma alta oscilação na resposta e o tempo de estabelecimento é lento, em torno de 60 segundos. O sistema realimentado apresenta ainda um erro estacionário.

Foi realizado o projeto de um controlador PID com o objetivo de melhorar o desempenho da resposta transitória, tornado-a mais rápida, e eliminar o erro de regime a resposta ao degrau (Ogata, 2010). Este controlador PID foi desenvolvido usando a abordagem analítica encontrada em (Ogata, 2010).

Sendo $G_{c}(s)$ a função de transferência do controlador PID e G(s) a função de transferência do Sistema de Dois Tanques em malha aberta, a função 
de transferência de malha fechada do Sistema de Dois Tanques com o controlador PID é dada por (4).

$$
G_{M F}(s)=\frac{G_{c}(s) G(s)}{1+G_{c}(s) G(s)}
$$

Desenvolvendo, temos (5).

$$
\frac{0,0194\left(K_{d} s^{2}+K_{p} s+K_{i}\right)}{s^{3}+\left(0,1312+0,0194 K_{d}\right) s^{2}+\left(0,0043+0,0194 K_{p}\right) s+0,0194 K_{i}}
$$

O sistema resultante é de terceira ordem, ou seja, possui três polos. Comparando esse sistema com um sistema de segunda ordem padrão com acréscimo de um polo, como pode ser visto em (6), é possível encontrar os valores das constantes do controlador em função de $\xi$ e $\omega_{n}$.

$$
\begin{gathered}
G_{d}(s)=\frac{\omega_{n}^{2}}{\left(s^{2}+2 \xi \omega_{\mathrm{n}} s+\omega_{n}^{2}\right)(s+p)} \\
=\frac{\omega_{n}^{2}}{s^{3}+\left(p+2 \xi \omega_{\mathrm{n}}\right) s^{2}+\left(2 \xi \omega_{\mathrm{n}} p+\omega_{n}^{2}\right) s+\omega_{n}^{2} p}
\end{gathered}
$$

Esse polo $\boldsymbol{p}$ inserido deve ser escolhido de forma que não altere a posição dos polos dominantes dos locais desejados. Com isso, as constantes do controlador PID, $K_{p}, K_{i}$ e $K_{d}$, podem ser encontradas comparando o denominador de $\mathrm{G}_{\mathrm{MF}}(\mathrm{s})$ com $\mathrm{G}_{\mathrm{d}}(\mathrm{s})$ resultando nas equações (7), (8) e (9) para realizar a alocação dos pólos desejados:

$$
\begin{gathered}
K_{p}=\frac{2 p \xi \omega_{\mathrm{n}}+\omega_{n}{ }^{2}-0,0043}{0,0194} \\
K_{i}=\frac{p \omega_{n}{ }^{2}}{0,0194} \\
K_{d}=\frac{p+2 \xi \omega_{\mathrm{n}}-0,1312}{0,0194}
\end{gathered}
$$

Neste projeto, foi considerado que os parâmetros do Sistema de Dois Tanques da Quanser são variáveis com variação de $10 \%$ em seus valores padrões para menos e para mais. Esses parâmetros são definidos com valores padrão $\mathrm{P} 1=0,0194, \mathrm{P} 2=$ 0,1312 e $\mathrm{P} 3=0,0043$, em intervalos, $[\mathrm{P} 1]=[0,01746$, $0,02134],[\mathrm{P} 2]=[0,11808,0,14432]$ e $[\mathrm{P} 3]=[0,00387$, $0,00473]$.

Os intervalos [P1], [P2] E [P3] são intervalos próprios, pois para qualquer valor dentro desses intervalos o sistema manterá uma resposta dentro dos critérios de desempenho desejado. As equações em função dos parâmetros P1, P2 e P3 podem ser vistas em (10), (11) e (12).

$$
\begin{gathered}
K_{p}=\frac{2 p \xi \omega_{\mathrm{n}}+\omega_{n}{ }^{2}-P 3}{P 1} \\
K_{i}=\frac{p \omega_{n}{ }^{2}}{P 1}
\end{gathered}
$$

$$
K_{d}=\frac{p+2 \xi \omega_{\mathrm{n}}-P 2}{P 1}
$$

Para requisitos do projeto foi considerado o tempo de estabelecimento de 30 segundos com critério de $5 \%$ e sobre-elevação máxima de $10 \%$. Com isso os valores de coeficiente de amortecimento e frequência natural não amortecida são $[\xi]=[\mathbf{0 , 8}$, $\mathbf{0 , 5 9}]$ e $\left[\omega_{n}\right]=[\mathbf{0 , 2}, \mathbf{0 , 1 6 9}]$, respectivamente, obtidos conforme visto em (Ogata, 2010).

Aplicando as propriedades e operações da aritmética intervalar modal encontradas em (Gardenes et al, 2001), (Prado, 2006) e (Group, 1998) às equações que determinam os valores das constantes do controlador PID, é possível encontrar os intervalos dos ganhos $\mathrm{K}_{\mathrm{p}}$, $\mathrm{K}_{\mathrm{i}}$ e $\mathrm{K}_{\mathrm{d}}$, conforme (13), (14) e (15).

$$
\begin{aligned}
& {\left[K_{p}\right]=\left[\frac{2 p \xi_{1} \omega_{\mathrm{n} 1}+\omega_{n 1}{ }^{2}-P 3_{2}}{P 1_{2}}, \quad \frac{2 p \xi_{2} \omega_{\mathrm{n} 2}+\omega_{n 2}{ }^{2}-P 3_{1}}{P 1_{1}}\right]} \\
& {\left[K_{i}\right]=\left[\frac{p \omega_{n 1}^{2}}{P 1_{2}}, \frac{p \omega_{n 2}^{2}}{P 1_{1}}\right]} \\
& {\left[K_{d}\right]=\left[\begin{array}{ll}
\frac{p+2 \xi_{1} \omega_{\mathrm{n} 1}-P 2_{2}}{P 1_{2}}, & \frac{p+2 \xi_{2} \omega_{\mathrm{n} 2}-P 2_{1}}{P 1_{1}}
\end{array}\right]}
\end{aligned}
$$

Sendo que $\boldsymbol{p}$ é um dos polos do sistema ao inserir o controlador PID, para esse polo determinou-se o valor de -0,022 para evitar valores altos de $K_{p}, K_{i}$ e $\mathrm{K}_{\mathrm{d}}$ que tornam a implementação do controlador inviável devido às limitações físicas do sistema. Além disso, esse valor cancela um zero produzido pelo PID. Isso evita que esse polo influencie no comportamento do sistema e permite que o controlador atue de acordo como projetado. Então, desenvolvendo as equações acima, os valores das constantes do controlador são: $\left[K_{p}\right]=[1,9827,1,6654], \quad\left[K_{i}\right]=$ $[0,0412,0,0360]$ e $\left[K_{d}\right]=[9,2634,5,9187]$.

As representações semânticas das equações apresentadas e suas interpretações são:

$$
\begin{gathered}
U\left(P 1 \in[P 1]^{\prime}\right) U\left(P 3,[P 3]^{\prime}\right) E\left(\omega_{n},\left[\omega_{n}\right]^{\prime}\right) \\
E\left(\xi,[\xi]^{\prime}\right) U\left(K_{p},\left[K_{p}\right]^{\prime}\right) K_{p}=\frac{2 p \xi \omega_{n}+\omega_{n}{ }^{2}-P 3}{P 1}
\end{gathered}
$$

Interpretação: Para todo valor de P1, para todo valor de P3, existe um valor de $\omega_{\mathrm{n}}$ e de $\xi$ para todo valor de $\mathrm{K}_{\mathrm{p}}$, onde todos estão dentro de seus respectivos intervalos, que satisfaz o predicado apresentado.

$U\left(P 1 \in[P 1]^{\prime}\right) E\left(\omega_{n},\left[\omega_{n}\right]^{\prime}\right) U\left(K_{i},\left[K_{i}\right]^{\prime}\right) K_{i}=\frac{p \omega_{n}{ }^{2}}{P 1}$

Interpretação: Para todo valor de P1, existe um valor de $\omega_{\mathrm{n}}$ para todo valor de $\mathrm{K}_{\mathrm{i}}$, onde todos estão dentro de seus respectivos intervalos, que satisfaz o predicado apresentado. 


$$
\begin{gathered}
U\left(P 1 \in[P 1]^{\prime}\right) U\left(P 2,[P 2]^{\prime}\right) E\left(\omega_{n},\left[\omega_{n}\right]^{\prime}\right) \\
E\left(\xi,[\xi]^{\prime}\right) U\left(K_{d},\left[K_{d}\right]^{\prime}\right) K_{d}=\frac{p+2 \xi \omega_{\mathrm{n}}-P 2}{P 1}
\end{gathered}
$$

Interpretação: Para todo valor de P1, para todo valor de $\mathrm{P} 2$, existe um valor de $\omega_{\mathrm{n}}$ e de $\xi$ para todo valor de $\mathrm{K}_{\mathrm{d}}$, onde todos estão dentro de seus respectivos intervalos, que satisfaz o predicado apresentado. Com o significado semântico proporcionado pelos Teoremas semânticos, pode-se garantir não apenas a estabilidade do sistema, mas também que para a planta tendo variação dentro dos intervalos dos parâmetros de incertezas estabelecidos pelo projeto, o sistema responderá satisfazendo aos intervalos das especificações para qualquer ganho de controlador dentro dos intervalos resultantes.

Com o controlador PID desenvolvido foram feitas algumas simulações e comparações e os resultados obtidos são apresentados no tópico Resultados.

\section{Resultados}

O primeiro teste realizado apresenta a resposta do sistema ao degrau unitário para três diferentes valores de parâmetros da planta. Os valores dos parâmetros da planta, P1, P2 e P3, foram variados de forma sincronizada para representar os dois extremos dos intervalos e o seu ponto médio. Os valores de $\mathrm{K}_{\mathrm{p}}, \mathrm{K}_{\mathrm{i}}$ e $K_{d}$ foram fixados em seus valores medianos. A Tabela 2 detalha os valores utilizados no teste.

Tabela 2. Especificações da simulação do Controlador PID.

\begin{tabular}{|c|c|c|c|c|c|c|}
\hline Sistema & $\mathbf{K}_{\mathbf{p}}$ & $\mathbf{K}_{\mathbf{i}}$ & $\mathbf{K}_{\mathbf{d}}$ & $\mathbf{P 1}$ & $\mathbf{P 2}$ & $\mathbf{P 3}$ \\
\hline sys_min & 1,82 & 0,038 & 7,59 & 0,0174 & 0,1180 & 0,0038 \\
\hline sys_medio & 1,82 & 0,038 & 7,59 & 0,0194 & 0,1312 & 0,0043 \\
\hline sys_max & 1,82 & 0,038 & 7,59 & 0,0213 & 0,1443 & 0,0047 \\
\hline
\end{tabular}

Os resultados obtidos podem ser vistos na Figura 2. Percebe-se que o erro em regime foi eliminado pelo controlador PID. Os requisitos de desempenho também foram atendidos obtendo sobre-elevação máxima inferior a $10 \%$ e tempo de estabelecimento menor que 30 segundos (com critério de 5\%).

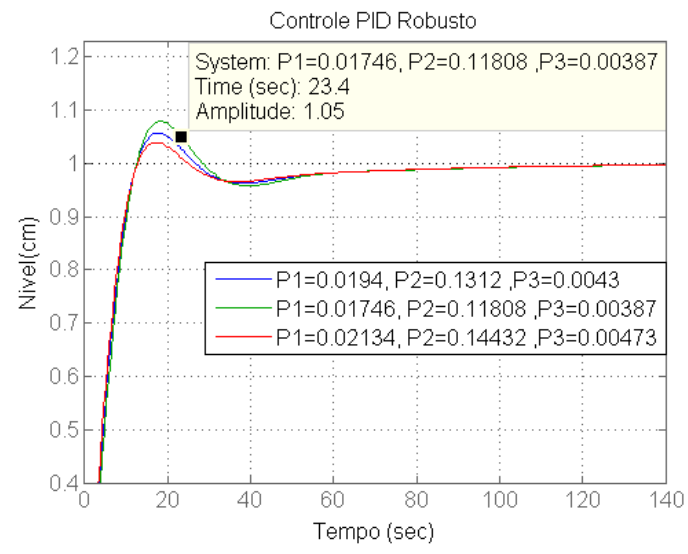

Figura 2. Resposta ao degrau unitário do sistema com o PID.
Foi desenvolvido um controlador PID tradicional (não robusto) para realizar comparações com o controlador robusto projetado. O controlador PID tradicional por alocação de pólos foi desenvolvido considerando os valores padrões da planta e tendo como requisitos máxima sobre-elevação de $10 \%$ e tempo de acomodação de 30 segundos. A Tabela 3 mostras os valores obtidos para $\mathrm{K}_{\mathrm{p}}, \mathrm{K}_{\mathrm{i}}$ e $\mathrm{K}_{\mathrm{d}}$ no projeto do controlador tradicional e os valores nos parâmetros da planta adotados para a simulação além dos valores atribuídos ao controlador PID robusto.

Tabela 3. Valores adotados na comparação do controlador Tradicional com o Robusto.

\begin{tabular}{|c|c|c|c|c|c|c|}
\hline Sistema & $\mathbf{K}_{\mathbf{p}}$ & $\mathbf{K}_{\mathbf{i}}$ & $\mathbf{K}_{\mathbf{d}}$ & $\mathbf{P 1}$ & $\mathbf{P 2}$ & $\mathbf{P 3}$ \\
\hline Tradicional & 1,47 & 0,032 & 4,65 & 0,0213 & 0,118 & 0,0038 \\
\hline Robusto & 1,82 & 0,038 & 7,591 & 0,0213 & 0,118 & 0,0038 \\
\hline
\end{tabular}

A eficácia do controlador robusto pode ser verificada na Figura 3. Essa simulação mostra o bom resultado do controlador PID Robusto em acompanhar o sinal de entrada quando comparado ao controlador tradicional que apresenta uma resposta indesejada para máxima sobre-elevação quando há variações nos parâmetros da planta. Enquanto o controlador robusto atende os requisitos do projeto com tempo de estabelecimento de 21,6 segundos e máxima sobre-elevação de $9,09 \%$, o controlador tradicional apresenta um tempo de estabelecimento de 23,7 segundos e máxima sobre-elevação de 10,4\%.

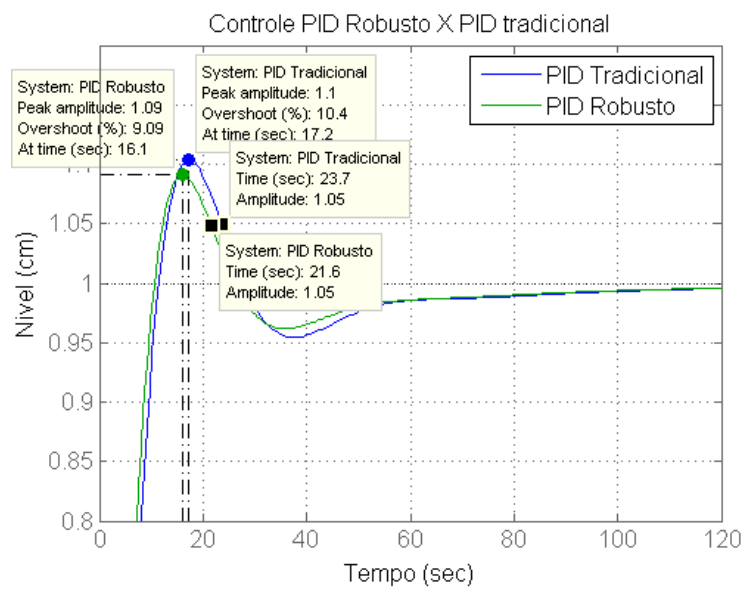

Figura 3. Comparação entre controlador Robusto e Tradicional.

Foram realizados ainda testes no Simulink de forma a simular o sistema de dois tanques o mais próximo possível da realidade, inserindo na simulação um bloco de saturação que implementa as limitações físicas do sistema. Essa simulação apresenta resultados ligeiramente diferentes aos obtidos por scripts do MATLAB, que modelam o sistema como se estes fossem ideais e livres de limitações físicas.

Através do Simulink é possível também aplicar outros sinais de entrada e com isso verificar como o sistema irá se comportar para diferentes entradas. Nas simulações anteriores o sinal de entrada aplicado era um degrau unitário o que provoca na saída um 
nível de água de $1 \mathrm{~cm}$ no segundo tanque do Sistema de Dois Tanques. Para verificar o funcionamento do sistema controlado para outros sinais de entrada aplicou-se um sinal degrau na entrada, porém com amplitude 10. É importante relembrar que o Sistema de Dois Tanques permite, em ambos os tanques, um nível máximo de $30 \mathrm{~cm}$. Os testes para essa entrada foram aplicados tanto no controlador PID Robusto quanto no controlador PID tradicional usando os mesmos valores de parâmetros da Tabela 3. Os resultados podem ser vistos na Figura 4.

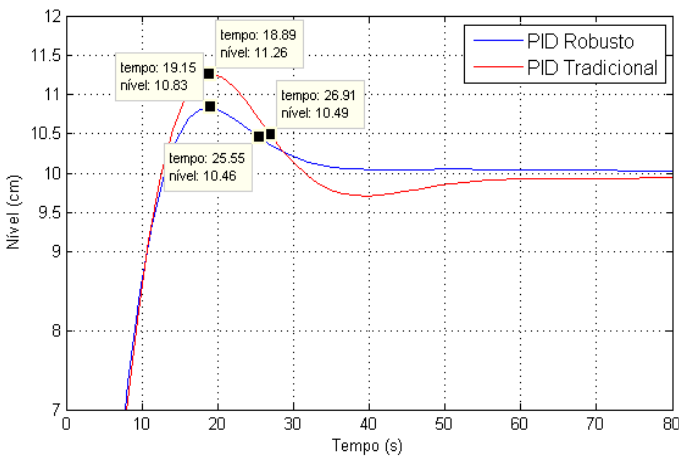

Figura 4. Resposta dos Controladores PID, Robusto e Tradicional para uma entrada com amplitude igual a 10.

Percebe-se pela Figura 4 que o controlador robusto continua gerando uma resposta dentro das especificações do projeto, com máxima sobreelevação de $8,3 \%$ e tempo de estabelecimento de 25,5 segundos, enquanto que o controlador tradicional apresenta uma oscilação indesejada com pico de 11,26 centímetros representando $12,6 \%$ de máxima sobre-elevação apesar de obter um tempo de acomodação de 26,9 segundos.

Nesta comparação torna-se claro que um controlador tradicional não é capaz de manter o desempenho desejado de um sistema quando este sofre variações em seus parâmetros. Sendo necessário, portanto, o projeto de um controlador robusto.

Foi analisado ainda o esforço de controle do sistema de forma a avaliar se o controlador desenvolvido provoca sinais de controle compatíveis com as limitações da planta. Sabe-se, por exemplo, que a máxima tensão que pode ser aplicada ao motor da planta é de 22 Volts estando, portanto, o esforço de controle limitado a esse valor. A Figura 5 mostra o esforço de controle gerado na execução da simulação anterior, exibida na Figura 4.

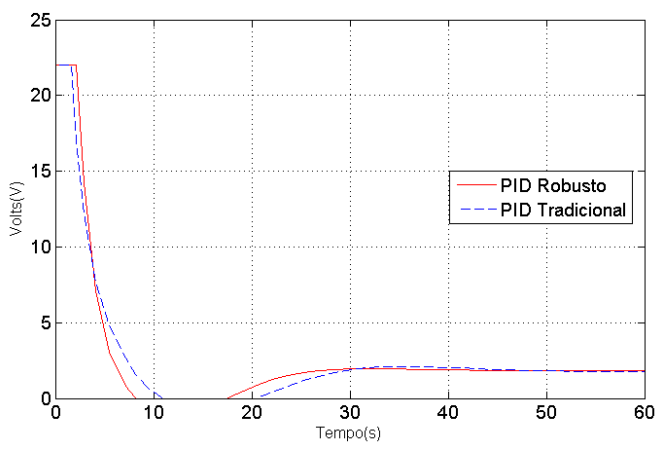

Figura 5. Esforço de Controle do Sistema.
Apesar da saturação que ocorre em alguns momentos nos sinais de esforço de controle a resposta do sistema não é comprometida, conseguindo ainda obter o desempenho desejado como foi observado na Figura 4. Assim, o projeto do controlador torna-se aplicável em situações reais.

\section{Conclusão}

Através dos resultados do controlador PID desenvolvido foi possível constatar que o erro em regime permanente foi eliminado além de o sistema obter o desempenho robusto desejado conforme os requisitos do seu projeto. Percebeu-se ainda que apesar do esforço de controle, em alguns momentos, chegar a saturar isso não impediu o sistema de ter o desempenho esperado pelo projeto.

O projeto de controladores robustos utilizando análise intervalar modal mostrou-se uma boa técnica de projeto de sistemas de controle, servindo de grande importância para sistemas que necessitem de garantia de desempenho mesmo que sujeito a variações nos seus parâmetros. Com isso, percebe-se que os resultados obtidos foram satisfatórios mostrando a importância deste trabalho e que o objetivo principal do projeto foi alcançado.

\section{Agradecimentos}

Agradecemos ao CNPq pela bolsa de Iniciação Científica concedida e à UEFS por todo suporte concedido para o desenvolvimento deste trabalho.

\section{Referências Bibliográficas}

Acióly, B. M (1991). Fundamentação computacional da matemática intervalar. Porto Alegre CPGCC da UFRGS.

Dorf, R. C. e Bishop, R. (2001). Sistemas de Controle Moderno. 8 ed, LTC.

Gardenes, E., Sainz, M. ., Jorba, L., Calm, R., Estela, R., Mielgo, H. and Trepat, A (2001). Modal intervals, Reliable Computing 7: 77-111. DOI: 10.1023/A:1011465930178

Group, S (1998). Extensiones de las funciones continuas, Report IMA 98-04-RR, Dept. de Informatica y Matematica Aplicada, Universidad de Girona, Spain.

Moore, R. E (1979). Methods and Applications of Interval Analysis, SIAM, Philadelphia. DOI: 10.1137/1.9781611970906

Ogata, K. (2010). Engenharia de Controle Moderno. 5 ed, Prentice Hall.

Prado, M. L. M (2006). Controle Robusto por Alocação de Pólos via Análise Intervalar Modal, Tese-Únicamp, Campinas.

Prado, M. L. M. e Paz, M. A (2008). Controle Robusto utilizando Estrutura PD e Análise intervalar Modal. In: XIII Congreso Latinoamericano de Control Automatico, Mérida.

Quanser (2004). Coupled Water Tank Experiments, Quanser, EUA. 